\title{
Intranasal Osteosarcoma in a Dog-A Case Report
}

\author{
Gabriela Noronha de Toledo, DVM, PhD ${ }^{\mathrm{a}, *}$, Pamela Rodrigues Reina Moreira, DVM, PhD ${ }^{\mathrm{b}}$, \\ Daniele Santos Rolemberg, DVM, MSc ${ }^{\mathrm{a}}$, Andréia Coutinho Facin, DVM, PhD ${ }^{\mathrm{a}}$, \\ Julio Edward Hough Monteiro, MSca ${ }^{a}$ Mareliza Possa de Menezes, DVM ${ }^{a}$, \\ Maria Eduarda Bastos Andrade Moutinho da Conceição, DVM, MSc ${ }^{a}$, \\ Felipe Augusto Ruiz Sueiro, DVM, $\mathrm{PhD}^{\mathrm{c}}$, Rosemeri de Oliveira Vasconcelos, DVM, PhD ${ }^{\mathrm{b}}$, \\ Paola de Castro Moraes, DVM, PhD ${ }^{\mathrm{a}}$, Mirela Tinucci Costa, DVM, PhD \\ Andrigo Barboza de Nardi, DVM, PhD
}

Keywords:

bone tumor

dog

neoplasia

veterinary pathology (source: $\mathrm{MeSH}$ )

${ }^{a}$ Department of Clinic and Veterinary Surgery, School of Agrarian and Veterinary Sciences, São Paulo State University (UNESP/FCAV), Jaboticabal, SP, Brazil

${ }^{b}$ Department of Veterinary Pathology, School of Agrarian and Veterinary Sciences, São Paulo State University (UNESP /FCAV), Jaboticabal, SP, Brazil

${ }^{c}$ VetPat-Laboratory of Veterinary Pathology and Molecular Biology, Campinas, SP, Brazil

\begin{abstract}
A B S T R A C T
Osteosarcoma is the most commonly reported bone tumor in dogs, typically affecting the axial and appendicular skeleton. Involvement of the paranasal sinuses and the nasal cavity is extremely rare. This report describes a 6-year-old, female, cross-breed dog presented with signs of 90 days duration and diagnosed with a mass located in the right nasal cavity. Radiography, rhinoscopy, and computed tomography findings suggested the presence of an intranasal tumor. Osteosarcoma in the nasal cavity was diagnosed based on histopathological examination and clinical and imaging findings. Survival time was 12 months from diagnosis.
\end{abstract}

(c) 2018 Elsevier Inc. All rights reserved.

\section{Introduction}

Osteosarcoma is a malignant mesenchymal tumor and is the most common primary bone tumor in dogs [1]. Intranasal tumors are considered quite unusual and comprise $1 \%-2 \%$ of all tumors in dogs [2,3]. Malignant tumors of epithelial origin (carcinomas) make up 70\% of intranasal tumors; with adenocarcinoma and squamous cell carcinoma being the most commonly reported, and sarcomas representing a smaller proportion [2-4]. Intranasal mesenchymal tumors consist of osteosarcoma, chondrosarcoma, fibrosarcoma, and undifferentiated sarcoma [4-6].

Osteosarcomas typically exhibit rapid local progression and mortality due to early lung metastasis. Metastasis rate for canine axial osteosarcomas is $11 \%-13 \%$ at the time of diagnosis and $35 \%$ $46 \%$ after diagnosis. However, it appears that the metastatic potential of canine axial osteosarcoma may vary according to the location of the tumor. Mandibular and maxillary osteosarcomas have lower metastatic potential than those in other sites. Higher metastatic rates have been described in osteosarcomas of rib, scapula, and skull [7].

In most cases, intranasal tumors are highly malignant, locally aggressive, and cause bone destruction in the early stages of the disease but usually have a low-metastatic rate [2]. Late in the course of the disease, metastasis to regional

\footnotetext{
* Corresponding author.

E-mail address: bitoledo@hotmail.com (G.N. de Toledo).
}

lymph nodes and lungs may occur in up to $40 \%$ of cases; however, death or euthanasia generally occurs as a result of clinical signs related to local primary disease rather than metastatic lesions [8].

Clinical signs associated with intranasal tumors consist of unilateral or bilateral nasal discharge (serous, mucoid, mucopurulent, or epistaxis), or a nonhealing ulcer on the nasal planum and deformation of the nasal planum and nose $[9,10]$. Less frequently, ocular discharge may be seen due to obstruction of the nasolacrimal duct by the nasal tumor [10]. Dogs with an intranasal mass may also experience discomfort on palpation of the nose and face and have sleeping disturbances ranging from restlessness to interrupted sleep due to obstructed breathing. Some dogs with neoplastic obstruction of the air passages do not adapt to open mouth breathing even at rest [10].

It is important to accurately define the extent of a nasal tumor and complete staging is recommended to guide treatment protocols and provide an accurate prognosis [2]. Computed tomography (CT) is an appropriate modality for investigation of intranasal tumors in dogs $[9,11,12]$. Specific relevant features to be evaluated on CT images are the integrity of the cribriform plate, involvement of the central nervous system, the degree of tumor invasion into surrounding tissues, and the morphology and anatomy of the skull structures $[4,9,10,12]$.

Treatment of nasal and intranasal tumors often includes radiation therapy, chemotherapy, and surgery, but the long-term prognosis is usually poor [2,3]. Radiation therapy is considered the best approach for intranasal tumors in dogs [3]. When used in 


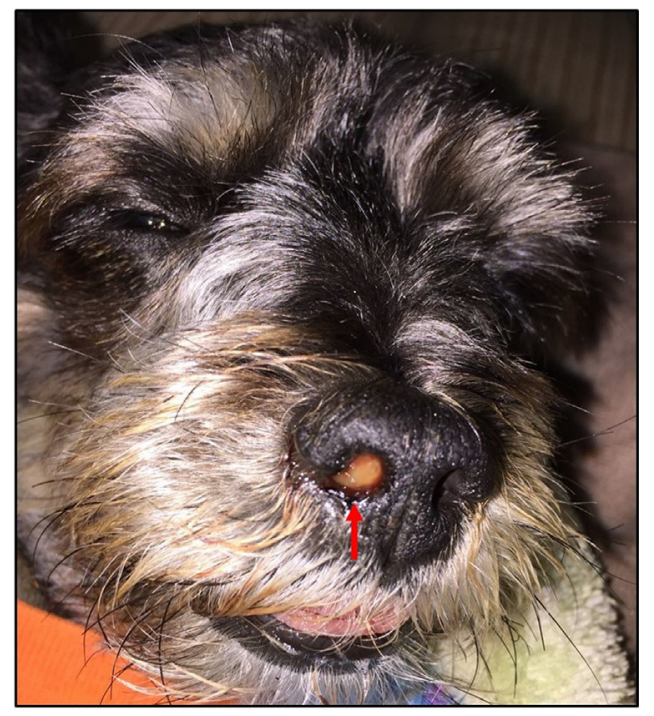

Fig. 1. Intranasal osteosarcoma in the 6-year-old dog described in this report. Note obstruction of right nasal passage by the tumor (red arrow), no facial asymmetry or swelling of the nasal planum was observed. The photograph was taken on the first appointment day.

combination with other treatment modalities, such as debulking surgery and chemotherapy [4], the reported survival time of dogs treated with radiotherapy protocols ranges from 8.5-19.7 months $[2,3,13]$.

A retrospective study described 17 cases of intranasal osteosarcoma over a 22-year period [5], demonstrating that in veterinary oncology, osteosarcoma in the nasal cavity is sporadic. Here we describe the imaging and histopathological features of this rare tumor and provide a brief literature review.

\section{Case History}

A 6-year-old, $6 \mathrm{~kg}$, sexually intact, female, cross-breed dog was admitted to the Veterinary Hospital of the School of Agrarian and Veterinary Sciences, São Paulo State University (FCAV/UNESP), with a history of 90 days of right unilateral epistaxis. The dog had previously undergone several clinical treatments for inflammatory diseases of the upper respiratory tract and for rickettsial disease. Epistaxis was initially reduced with 28-day antimicrobial treatment; however, a relapse occurred when therapy was withdrawn, after which time the epistaxis became progressively more severe.

On first examination there was moderate pain on palpation of the nasal cavity, unwillingness to open the mouth, and cyanosis of the oral mucosa. There were no visible changes to or deformation of the nose and no facial asymmetry was observed (Fig 1).

\section{Results}

Further examinations were performed to rule out systemic abnormalities. Blood samples were submitted for blood count and biochemistry analysis, but all measured parameters were within normal ranges. Abdominal ultrasonography and neurological examination revealed no abnormalities. Urinalysis was unremarkable. Thoracic radiographs in projections (right and left lateral and ventrodorsal) were performed and no significant abnormalities were observed.
Skull CT, centered on the nasal cavity was performed with a GE Hispeed FX1 Helicoidal. Transverse slices of $3 \mathrm{~mm}$ thickness were obtained and analyzed in soft tissue $(\mathrm{W}=1500 / \mathrm{L}=300)$ and bone windows ( $\mathrm{W}=40 / \mathrm{L}=350$ ). Postcontrast images were obtained 40 seconds after administration of Ioexol $(1.5 \mathrm{mg} / \mathrm{kg}) \mathrm{IV}$. Multiplanar reconstructions were also obtained.

A large amorphous, poorly rim-enhancing soft-tissue structure was visible at the dorsolateral edge of the right nasal cavity, identified on postcontrast transverse images, at the first premolar level (Fig 2). The mass obliterated the entire right nasal cavity and extended from the nostril rostrally to the right nasal sinus. The mass was invading the nasopharyngeal meatus and pterygopalatine fossae, displacing the right ocular globe dorsolaterally. There was extensive turbinate osteolysis, dorsolateral to the right maxilla, with full-thickness pterygoid and palatine bone destruction that resulted in ventral bulging of the hard palate. There was also significant lysis of the lacrimal and sphenoid bones, as well as invasion of the orbital cavity by the tumor.

On caudal and reconstructed dorsal plane images at the orbit level, the right frontal sinus was filled by a homogeneous and soft-tissue attenuating expansile mass. Significant osteolysis and loss of definition of the cribriform plate was also apparent, suggesting that the mass could be invading the rostral encephalic cavity (Fig 2).

Rhinoscopy revealed a large reddish mass, with a smooth external surface and soft consistency, occupying the right nasal passage. Multiple biopsy specimens were obtained; although histopathologic examination was inconclusive-revealing dysplasia and atypical nasal epithelium.

Due to a worsening of clinical signs, nasal sinus debulking surgery was performed 30 days later. At surgery the mass was observed to have invaded through the right lateral portion of the nasal sinus. Samples collected for bacterial culture resulted in no growth. All the collected material, including bone fragments, was sent for a second histopathological examination.

Histopathological evaluation indicated neoplastic proliferation of poorly differentiated mesenchymal cells. The sample was highly cellular and consisted of a poorly demarcated, unencapsulated mass with an infiltrative growth pattern, and nuclear pleomorphism.

Bone matrix production by tumor cells was observed along with multiple foci of osteoid mineralization. Furthermore, multiple mitotic figures (30), wide and vacuolated cytoplasm, and nuclear alterations (anisokaryosis, anisocytosis, and karyomegaly) were also seen. The nuclei were elongated and hyperchromatic, with densely clustered chromatin and prominent nucleoli (Fig 3). The histopathological diagnosis was osteosarcoma.

Palliative radiotherapy and tyrosine kinase inhibitors were recommended as treatment but were declined by the owner on the grounds of cost. The dog then received the following treatments: adjuvant chemotherapy (alternating the drugs every 3 weeks: doxorubicin $30 \mathrm{mg} / \mathrm{m}^{2} / \mathrm{IV}$ and carboplatin $300 \mathrm{mg} / \mathrm{m}^{2} / \mathrm{IV}$ ), metronomic chemotherapy (piroxicam $0.3 \mathrm{mg} / \mathrm{kg} / \mathrm{d} / \mathrm{PO} / 6$ months and cyclophosphamide $12.5 \mathrm{mg} / \mathrm{m}^{2} / \mathrm{d} / \mathrm{PO}$ ) and routine follow-up examinations were recommended.

After 6 adjuvant chemotherapy sessions alternating doxorubicin and carboplatin, the dog was again presented with an obstruction of the right nasal cavity and underwent a second debulking surgery to relieve signs. At that time, adjuvant chemotherapy was discontinued, and only palliative pain relief was continued. After 3 months, a third debulking surgery was performed to relieve recurring signs.

Thoracic radiographs and ultrasonography over 12 months failed to identify any metastatic disease. 


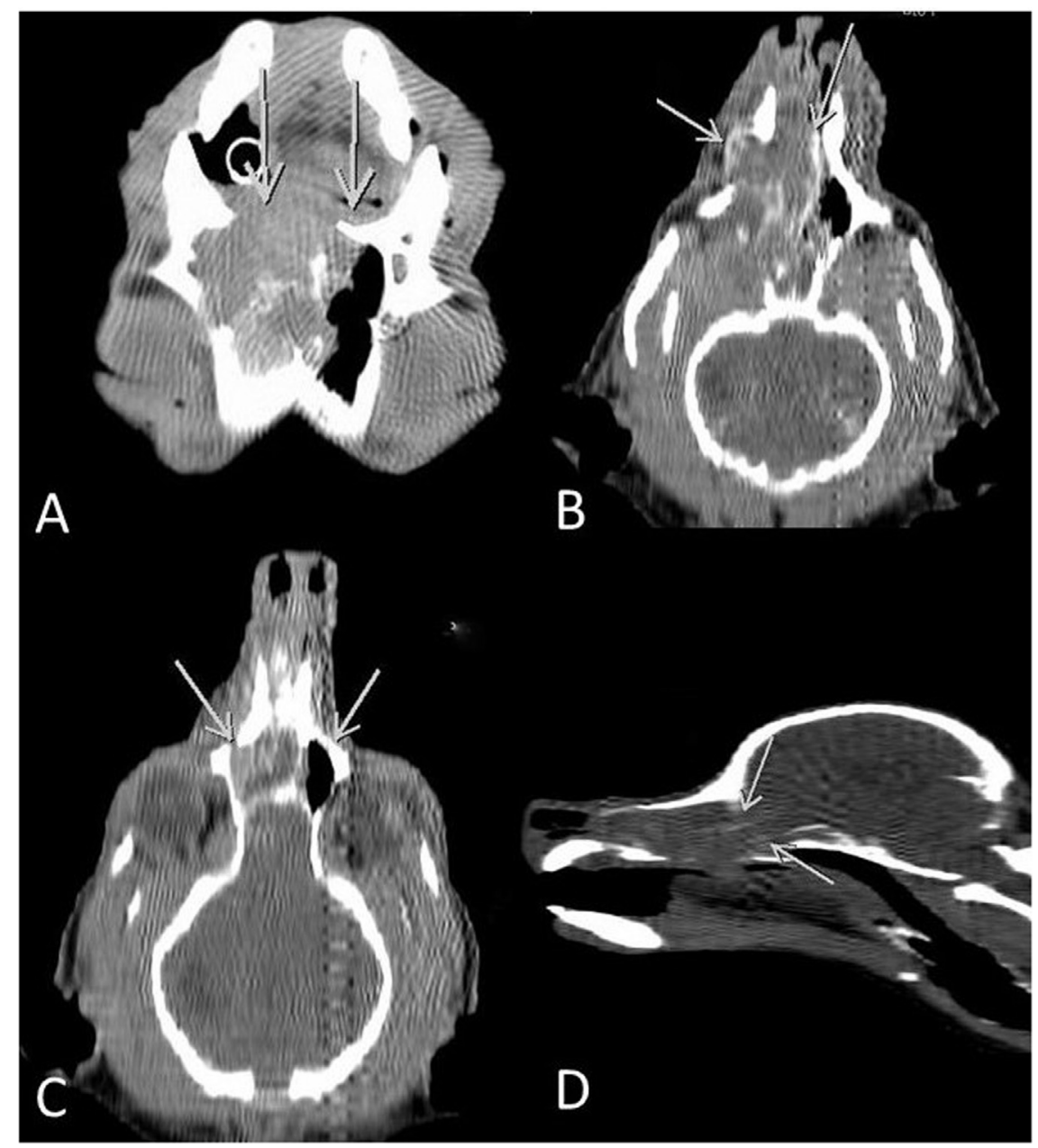

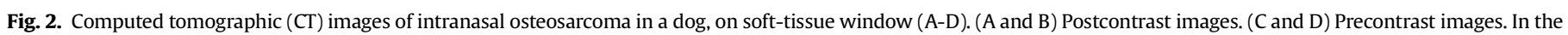

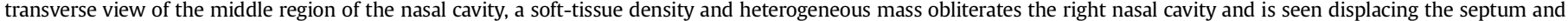

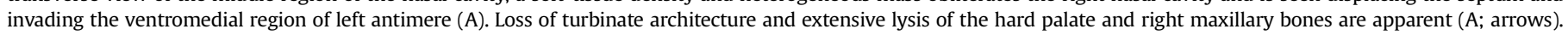

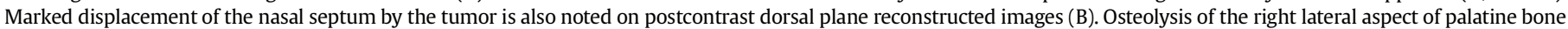

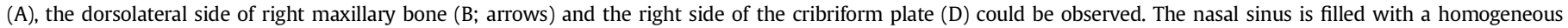

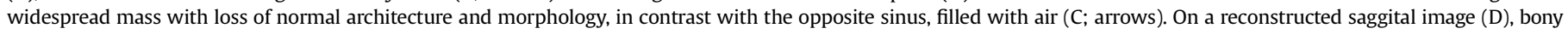
boundaries between nasal the cavity and cranial vault are lacy in appearance suggesting lysis of the cribriform plate (arrows).

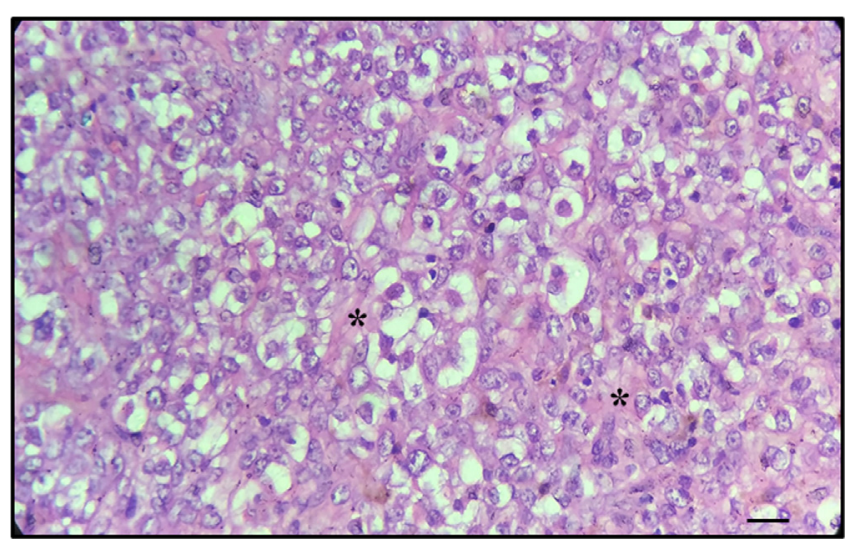

Fig. 3. Photomicrograph of a sample of canine bone tissue showing neoplastic mesenchymal proliferation from a 6-year-old female dog with intranasal osteosarcoma. There are areas of cartilage with eosinophilic deposits of osteoid $\left({ }^{*}\right)$. Bar is $20 \mu \mathrm{m}$, hematoxylin and eosin.

\section{Discussion}

The median age of dogs diagnosed with an intranasal tumor is 8.75 years (range: $1.75-22$ years) [3]. In agreement with the previous study, this report describes a dog diagnosed at 6 years of age.

Nasal discharge is a common clinical sign in dogs with chronic nasal disease. Differential diagnoses for dogs with chronic unilateral or bilateral epistaxis, facial asymmetry, and exophthalmos include nasal tumors, chronic inflammatory rhinitis, and aspergillosis [14]. In this case report, the signs demonstrated by the patient were unilateral epistaxis, moderate pain, and cyanosis. All these signs were attributed to upper airway obstruction. Although the clinical signs in this dog were most consistent with neoplasia, definitive diagnosis requires the histopathologic evaluation of a biopsy specimen.

This report reaffirms the importance of complementary examinations, specifically, CT and histopathology. The patient, in this case, exhibited a prolonged period of epistaxis but did not show 
any outer deformation of the nasal cavity. It is important to note the significance of clinical signs and detailed anamnesis; however, the complementary examinations are important for establishing differential diagnoses. In this case, the patient received several different treatments for a number of months, and delayed diagnosis may reduce survival times in intranasal tumors.

Osteosarcomas may vary widely in their histological appearance, but in all cases a definitive diagnosis is based on the production of osteoid or bone by malignant mesenchymal cells [15]. Because of the multipotential nature of primitive mesenchymal cells, the tumor matrix may contain varying amounts of cartilage, collagen, and osteoid, but even in tumors where the cartilage component predominates, the presence of osteoid denotes a diagnosis of osteosarcoma [15]. The presence of osteioid reflects the greater malignant potential of mesenchymal tumors with osteogenic potential. Bone formation also occurs in some chondrosarcomas, but indirectly through the endochondral ossification of the tumor cartilage. The bones thus formed should not be confused with the tumor bone [15]. Osteosarcoma was diagnosed in this case on the basis of samples containing only basophilic bone tissues with osteoid formation and no morphological structures characterizing the presence of chondroid matrix.

In a study describing the survival time of different types of intranasal sarcomas in dogs, the median survival was 444 days (range: 7-1936 days) when submitted to radiotherapy [3]. Specifically, the median survival time of intranasal osteosarcoma was 624 days (range: 301-1185 days) [3], and the survival time in dogs with intranasal osteosarcoma is generally longer than for other types of intranasal sarcoma because osteosarcoma develops more slowly and is more sensitive to radiation therapy [3]. Without treatment, the survival time for this type of tumor in dogs is less than 77 days [19]. Although the survival time in this case was short compared to times reported in the veterinary literature for intranasal osteosarcoma, quality of life was well maintained.

In one study, the median survival time of dogs with intranasal sarcomas (chondrosarcoma (42), undifferentiated sarcoma (22), fibrosarcoma (12), osteosarcoma (6), myxosarcoma (2), hemangiosarcoma (1), liposarcomas, and (1) receiving radiotherapy was evaluated. In addition, median survival was compared between different treatment modalities. Totally, 86 dogs were evaluated and 8 received cytoreductive surgery, 11 received multiple chemotherapy protocols, and 4 had a combination of surgery and chemotherapy [3]. The median survival time for dogs receiving surgery was 1185 days, chemotherapy was 376 days, and for combined surgery and chemotherapy treatment was 344 days [3].

Previous studies have concluded that cytoreductive surgery alone does not increase survival time $[2,16,17]$. Another study evaluated the median survival time of dogs with intrasanal tumors divided into groups by histological diagnosis (carcinomas, sarcomas, and osteosarcomas). In this study, the dogs diagnosed with intranasal osteosarcomas were treated by stereotactic radiosurgery and survival of 100 days was reported [13]. In this case report, the patient had 3 debulking surgeries and adjuvant chemotherapy and the survival time was 360 days or 12 months. Therefore, future investigations of dogs with intranasal osteosarcoma are needed to define an accurate survival time. This patient maintained a good quality of life with relief from clinical signs for 1 year. Over a period of 6 months, the patient had three debulking surgeries as palliative treatment. It should be emphasized that at each tumor recurrence, especially in a short period of time, the tumor usually shows increasingly aggressive biologic behavior.

Lesions within the nasal cavity are easily accessed through the nares, can be visualized through a rigid endoscope, and can be biopsied using endoscopic forceps in an operative cannula channel [4]. Only small biopsy specimens can be obtained with this method and there is a risk of collecting nondiagnostic specimens. Large tissue samples can be obtained blindly with glass biopsy forceps [4]. The depth to which the caliper should be inserted into the nasal cavity is guided by CT images [4]. If the tumor has eroded through the dorsal or lateral wall of the nasal cavity, a small incision in the skin may be made over the area of interest and a sample of tissue collected [4]. If the tumor is located in the frontal sinus and only extends marginally into the nasal cavity, then the frontal sinus can be trephined, and cup biopsy forceps used to collect tissue samples [4]. If the tumor is in the caudal nasal cavity, samples can be collected with the help of a flexible endoscope that is retroflexed into the nasopharynx [4]. Open rhinotomy can provide large, good quality biopsy specimens; however, complications from the procedure, such as subcutaneous emphysema and hemorrhage, are common [18]. In the present case, the first attempt to collect material for biospy was through rhinoscopy and the result was inconclusive. The second sample was collected by trepanation, and complications were not observed.

Chemotherapy can be used as primary therapy or adjuvant therapy and complete remission is unlikely when chemotherapy is used as a sole modality, and survival times are reduced $[3,4]$. However, the alternating administration of doxorubicin and carboplatin together with piroxicam can result in complete or partial remission for some types of intranasal tumors, such as adenocarcinomas, osteosarcomas, squamous cell carcinoma, transitional cell carcinoma, and undifferentiated cell carcinoma [8]. In this case, the patient had to undergo 3 debulking surgeries to palliate the signs during the survival time suggesting that the chemotherapy was not effective.

In veterinary oncology, tyrosine kinase inhibitors (TKI) have been developed as antiangiogenic agents based on their inhibition of vascular endothelial growth factor receptor and platelet-derived growth factor receptor, 2 receptor families known to play an important role in tumor angiogenesis. These inhibitors have been used as antiangiogenic agents and have biological activity in a wide range of tumors with no obvious mutations including carcinomas, sarcomas, mast cell tumors, melanomas, and lymphomas [20]. In a study involving different tumors (carcinomas, sarcomas, mast cell tumors, melanomas, and lymphomas), 57 dogs were treated with TKI. Objective responses were observed and documented in 16 dogs for a response rate of $28 \%$. Stable disease for $>10$ weeks was observed in 15 additional dogs, for a resultant biological activity of $54 \%$. This study provided the first evidence that multisegmented TKI may exhibit broad activity against a variety of spontaneous malignant tumors in patients with canine cancer [21]. The client in this case declined the tyrosine kinase treatments due to the high cost of the medications.

Prognostic parameters have been previously described for dogs with intranasal tumors. Parameters associated with longer survival time include dogs less than 10 years of age at diagnosis, dogs without regional lymph node or lung metastasis, dogs with lower stage disease, and dogs treated with orthovoltage x-rays [22]. In one veterinary study, there were no cases of lymph node metastasis at the time of diagnosis which suggests that the incidence of lymph node metastasis in canine intranasal sarcomas is low [3]. During follow-up, monthly thoracic radiographs and regional lymph node cytology were performed without evidence of metastatic disease. Our findings were in line with previous reports regarding metastasis in the local lymph node and lungs.

Regardless of the treatment chosen, early diagnosis is an essential prognostic factor and improves the outcome of patients with intranasal osteosarcoma. However, many tumors present with aggressive features and unfavorable biological behavior and regardless of the treatment modalities selected, therapy may not be effective. 


\section{Conclusion}

Despite their low frequency, intranasal osteosarcoma and other intranasal tumors are potential causes of chronic nasal discharge, epistaxis, and nasal obstruction, especially in dogs over 6 years. These clinical signs should be thoroughly investigated because diagnoses other than nasal tumors eg inflammatory or infectious diseases may have a favorable prognosis. However, the prognosis for dogs with intranasal tumors is usually poor.

\section{References}

1. Szewczyk M, Lechowski R, Zabielska K. What do we know about canine osteosarcoma treatment?-review Vet Res Commun 39:61-67, 2015

2. Dycus DL, Akin EY, Crabtree AC, McEwen VK. What is your neurologic diagnosis? Intranasal osteosarcoma J Am Vet Med Assoc 237:37-39, 2010

3. Sones E, Smith A, Schleis S, et al. Survival times for canine intranasal sarcomas treated with radiation therapy: 86 cases (1996-2011). Vet Radiol Ultrasound 54:194-201, 2013

4. Weeden AM, Degner DA. Surgical approaches to the nasal cavity and sinuses. Vet Clin Small Anim 46:719-733, 2016

5. Patnaik AK, Lieberman PH, Erlandson RA, et al. Canine sinonasal skeletal neoplasms: chondrosarcomas and osteosarcomas. Vet Pathol 21:475-482, 1984

6. Patnaik AK. Canine sinonasal neoplasms: clinicopathological study of 285 cases. J Am Anim Hosp Assoc 25:103-113, 1989

7. Farcas N, Arzi B, Verstraete FJ. Oral and maxillofacial osteosarcoma in dogs: a review. Vet Comp Oncol 12:169-180, 2014

8. Langova V, Mutsaers AJ, Phillips B, Straw R. Treatment of eight dogs with nasal tumors with alternating doses of doxorubicin and carboplatin in conjunction with oral piroxicam. Aus Vet J 82:676-680, 2004

9. Worley DR. Nose and nasal planum neoplasia, reconstruction. Vet Clin North Am Small Anim Pract 46:735-750, 2016
10. Lux CN, Culp WTN, Johnson LR, et al. Prospective comparison of tumor staging using computed tomography versus magnetic resonance imaging findings in dogs with nasal neoplasia: a pilot study. Vet Radiol Ultrasound 58:315-325, 2017

11. Meler E, Dunn M, Lecuyer M. A retrospective study of canine persistent nasal disease: 80 cases (1998-2003). Can Vet J 49:71-76, 2008

12. Drees R, Forrest LJ, Chappell R. Comparison of computed tomography and magnetic resonance imaging for the evaluation of canine intranasal neoplasia. $J$ Small Anim Pract 50:334-340, 2009

13. Kubicek L, Milner R, An Q, et al. Outcomes and prognostic factors asssociated with canine sinonasal tumors treated with curative intent cone-based stereotactic radiosurgery (1999-2013). Vet Radiol Ultrasound 57:331-340, 2016

14. Fromberg LM, Morandi F, Lusby AL. What is your diagnosis? Osteosarcoma in a dog J Am Vet Med Assoc 236:1179-1180, 2010

15. Thompson KG, Dittmer KE. Tumor of bones. In: Meuten DJ, editor. Tumors in Domestic Animals. 5th ed. Iowa: Ames; 2017. p. 356-424

16. Adams WM, Bjorling DE, McAnulty JE, Green EM, Forrest LJ, Vail DM. Outcome of accelerated radiotherapy alone or accelerated radiotherapy followed by exenteration of the nasal cavity in dogs with intranasal neoplasia: 53 cases (1990-2002). J Am Vet Med Assoc 15:936-941, 2005

17. Laing EJ, Binnington AG. Surgical therapy of canine nasal tumors: A retrospective study (1982-1986). Can Vet J 29:809-813, 1988

18. Kuhlman GM, Taylor AR, Thieman-Mankin KM, Griffin J, Cook AK, Levine JM. Use of a frameless computed tomography-guided stereotactic biopsy system for nasal biopsy in five dogs. J Am Vet Med Assoc 15:929-934, 2016

19. Morris JS, Dunn KJ, Dobson JM, White RAS. Effects of radiotherapy alone and surgery and radiotherapy on survival of dogs with nasal tumours. J Small Anim Pract 35:567-573, 1994

20. London C, Mathie T, Stingle N, et al. Preliminary evidence for biologic activity of toceranib phosphate (Palladia(®) in solid tumours. Vet Comp Oncol 10:194-205, 2012

21. London CA. Tyrosine kinase inhibitors in veterinary medicine. Top Companion Anim Med 24:106-112, 2009

22. LaDue TA, Dodge R, Page RL, Price GS, Hauck ML, Thrall DE. Factors influencing survival after radiotherapy of nasal tumors in 130 dogs. Vet Radiol Ultrasound 40:312-317, 1999 